\title{
Suppression of Guard-Trace Resonance by Matched Termination for Reducing Common-Mode Radiation
}

\author{
Tetsushi WATANABE $^{\dagger \text { a) }}$, Tohlu MATSUSHIMA ${ }^{\dagger \dagger}$, Yoshitaka TOYOTA $^{\dagger \dagger}$, Osami WADA $^{\dagger \dagger}$, Members, $^{\circ}$ \\ and Ryuji KOGA ${ }^{\dagger \dagger \dagger}$, Fellow
}

\begin{abstract}
SUMMARY We propose a novel technique of matching at both ends of the guard trace to suppress resonance. This approach is derived from the viewpoint that the guard trace acts as a transmission line. We examined that matched termination suppresses guard-trace resonance through simulating a circuit and measuring radiation. We found from these results that the proposed method enables guard-trace voltages to remain low and hence avoids increases in radiation. In addition, we demonstrated that "matched termination at the far end of the guard trace" could suppress guard-trace resonance sufficiently at all frequencies. We eventually found that at least two vias at both ends of the guard trace and only one matching resistor at the far end could suppress guard-trace resonance. With respect to fewer vias, the method we propose has the advantage of reducing restrictions in the printed circuit board layout at the design stage.

key words: printed circuit board, common mode radiation, guard trace, imbalance difference model, current division factor
\end{abstract}

\section{Introduction}

Common-mode radiation is a major factor in electromagnetic interference (EMI) from printed circuit boards (PCBs) [1]. High-speed signal traces running either above a narrow return plane or close to the edge of a return plane cause common-mode radiation. To suppress EMI below a prescribed level, a scheme for controlling EMI should be deployed at the PCB design stage [2]. For this reason, we developed an "imbalance difference model" [3], which was specialized for quantitatively and quickly estimating common-mode radiation.

To test and corroborate the efficiency of the model, we estimated what effect a guard trace would have on commonmode radiation [4], [5]. A guard trace running along the signal line is commonly used for enlarging the path of the return current and reducing common-mode radiation from PCBs [6]-[8]. However, a guard trace that can reduce common-mode radiation is difficult to design because we need to use a 3-D full-wave electromagnetic simulator. In contrast, we demonstrated through 2-D static electric-field simulation with the imbalance difference model, which esti-

\footnotetext{
Manuscript received November 1, 2009.

Manuscript revised March 4, 2010.

The author is with Industrial Technology Center of Okayama Prefecture, Okayama-shi, 701-1296 Japan.

${ }^{\dagger}$ The authors are with the Graduate School of Engineering, Kyoto University, Kyoto-shi, 615-8510 Japan.

${ }^{\dagger \dagger}$ The authors are with the Graduate School of Natural Science and Technology, Okayama University, Okayama-shi, 7008530 Japan.

a)E-mail: watanabe@okakogi.go.jp

DOI: 10.1587/transcom.E93.B.1746
}

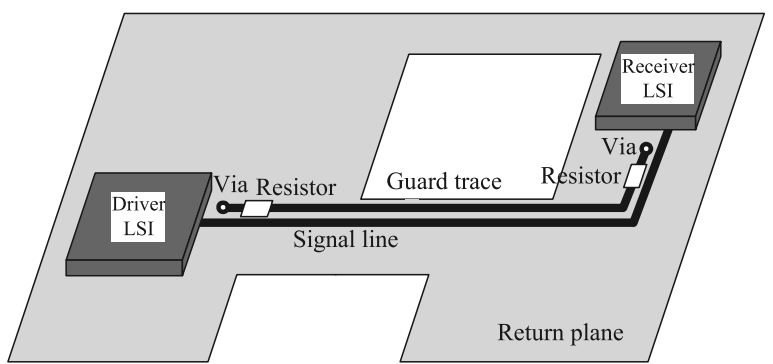

Fig. 1 Guard trace with termination resistors, placed near signal line in PCB.

mated within an error of $3 \mathrm{~dB}$, that common-mode radiation was reduced by placing the guard trace [5].

To confirm the advantages of the imbalance difference model, we have already proposed a method of design to reduce the number of vias in the guard trace by using the model [9]. A guard-trace generally needs many via connections to the return plane with short intervals. This is because a guard trace with long via intervals will generate resonance and hence increase common-mode radiation at frequencies of interest [10]. To reduce the number of vias in the guard trace by using the model predicted that the guard trace should be connected to the return plane only at the location where the cross-sectional structure of the transmission line changes according to the imbalance difference model [9]. As a result, they demonstrated that the common-mode excitation of the guard-trace voltage was eliminated and hence common-mode radiation was reduced even when the guard trace resonated.

In this paper, we propose a technique to suppress the guard-trace resonance itself so as not to increase the guardtrace voltage. From the viewpoint that the guard trace acts as a transmission line, matching both ends of the guard trace should help to suppress resonance. Placing matching resistors (Fig. 1) close to the vias, we investigated what effect they would have on reducing common-mode radiation. The vias were eventually reduced to two located at both ends of the guard trace. This technique should help avoid the restrictions caused by vias when designing multilayered boards.

\section{Suppression of Guard-Trace Resonance}

\subsection{Guard-Trace Resonance and Increased Radiation}

Test boards with a partially narrow return plane were used 


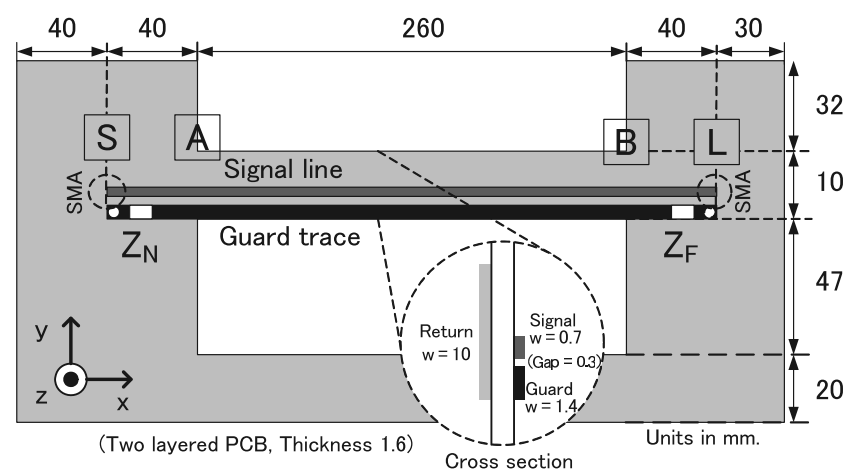

Fig. 2 Test board with signal line and guard trace.

in our previous work [4], [5]. This was because commonmode radiation increases with a narrow return plane or a signal trace placed near the return-plane edge. We also used a test board in this research that had a similar structure to that used in the previous work to investigate common-mode radiation.

Figure 2 shows a test board consisting of two layers: the top layer is for a signal line and a guard trace and the bottom layer is for the return plane. An SMA connector was installed at each end of the signal line from the back of the board. The signal was fed at point $\mathrm{S}$ and the load was connected at point $\mathrm{L}$. The characteristic impedance of the signal line was approximately $75 \Omega$.

The guard trace was placed near the signal trace because it plays the role of a return current path in suppressing common-mode radiation. Two termination resistors, $Z_{\mathrm{N}}$ and $Z_{\mathrm{F}}$, which denote near-end and far-end termination impedances, respectively, were placed at both ends of the guard trace. Another test board without a guard trace was also fabricated as a reference.

According to Ref. [5], the reduction in common-mode radiation from a test board with a guard trace to the reference board was estimated to be $8.0 \mathrm{~dB}$. This estimate was based on the imbalance difference model [3]. This model explains the mechanism for common-mode excitation when a common-mode voltage source is generated, where the crosssectional structure of the transmission line changes. Therefore, voltage sources generate at points A and B in the test board in Fig. 2. The magnitude of each source can be calculated with the cross-sectional dimensions [11].

Figure 3(a) plots the measured results of radiation from the test board $\left(Z_{\mathrm{N}}=Z_{\mathrm{F}}=0 \Omega\right)$ and the reference board. The radiation is reduced due to the guard trace except for a few frequencies. No reduction was observed at $500 \mathrm{MHz}$, and furthermore, increases in radiation were observed at around $750 \mathrm{MHz}$ and $1 \mathrm{GHz}$ even with the guard trace in place.

Figure 3(b) plots the guard-trace voltage at point B. The guard-trace voltage takes a large value at the same frequencies that no reduced radiation was observed.

A transmission line with short termination at both ends resonates at $n \lambda / 2(n=1,2 \ldots)$ along the length of the line, where $\lambda$ is the wavelength. Then, the resonant frequencies,

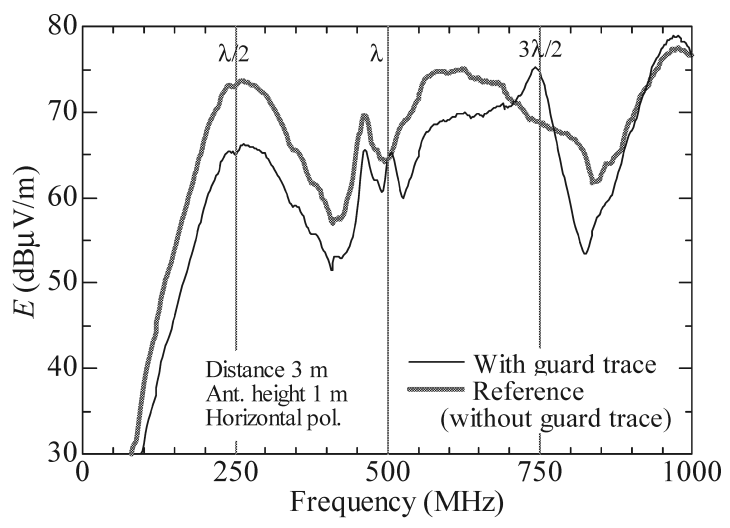

(a) Measured radiation.

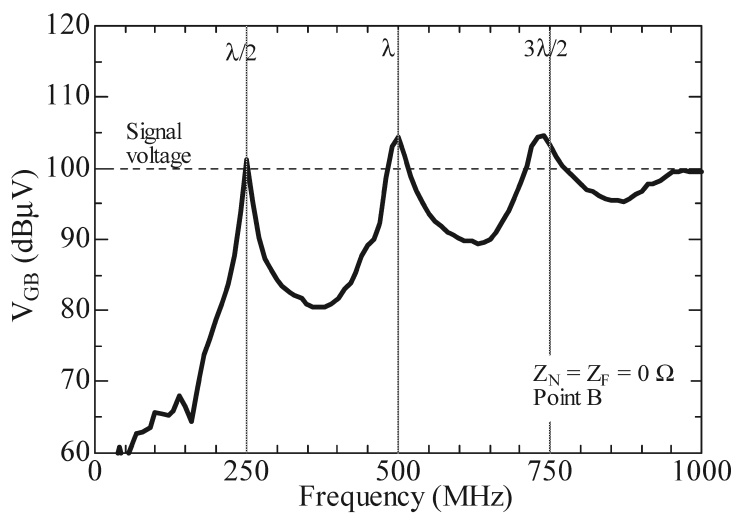

(b) Measured guard-trace voltage at point B.

Fig. 3 Increased radiation due to guard-trace resonance.

$f_{n}$, are obtained by

$$
f_{n}=\frac{n}{2 L} \frac{c_{0}}{\sqrt{\varepsilon_{\mathrm{eff}}}}(n=1,2, \ldots)
$$

where, $L, c_{0}$, and $\varepsilon_{\text {eff }}$ are the length of line, the velocity of light in vacuum and the effective relative permittivity, respectively. In the test board shown in Fig. 2, where $L=$ $340 \mathrm{~mm}$ and $\varepsilon_{\mathrm{eff}}=3.12$, the resonant frequencies are multiples of $250 \mathrm{MHz}$. At these resonant frequencies, the guardtrace voltage is increased as seen in Fig. 3(b).

\subsection{Proposed Method of Suppressing Guard-Trace Reso- nance}

This section explains the method of suppressing guard-trace resonance with termination resistors.

Figure 4(a) outlines the mechanism for guard-trace resonance in a standard case when vias are placed at both ends of the guard trace. The signal trace and the guard trace are regarded as asymmetrically coupled transmission lines. The source power exciting the guard trace is crosstalk from the signal line. When the electromagnetic power reaches one end of the guard trace, all the power is reflected because the via at the end acts as a short termination. The same phenomenon occurs at the other end. Then, multiple reflections lead to resonance and generate large guard-trace voltages, 


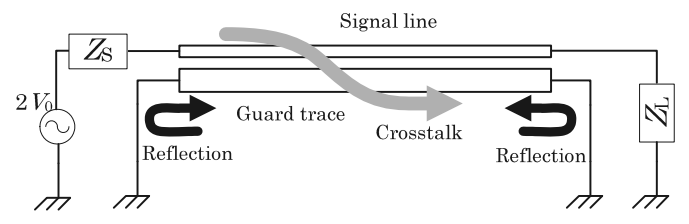

(a) Standard case of guard trace (without termination resistors)

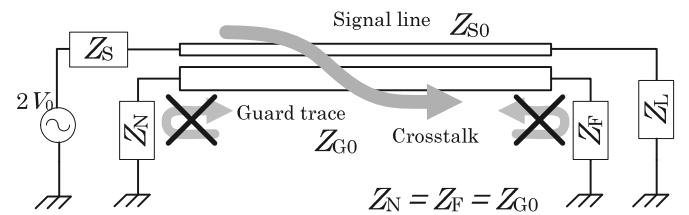

(b) Guard trace with matched terminations.

Fig. 4 Mechanism for guard-trace resonance and method of suppression

as shown in Fig. 3(b).

Let us now consider when matched resistors are connected at both ends of the guard trace, as shown in Fig. 4(b). In this case, the power in the guard trace is not reflected but absorbed at the termination. Therefore, large guard-trace voltages with multiple reflections do not generate at any frequencies. This leads to the elimination of vias in the guard trace.

Furthermore, the near-end resistor should be removed for the following reasons. Most of coupled power is absorbed at the far-end termination because the far-end crosstalk between coupled microstrip lines is generally larger than near-end crosstalk. Therefore, removing the near-end resistor (short end) will not greatly increase guardtrace voltage. Placing a matched resistor at only the far end will eventually be a cost-effective solution. In the following sections, we explain how we tested and verified that guard-trace voltage was reduced without increasing common-mode radiation.

\subsection{Equivalent Circuit Model for Calculating Guard-Trace Voltage}

According to our previous paper [9], the guard-trace voltage at a discontinuous point of the cross section generates another common-mode voltage source (see Appendix). The voltage is given as:

$$
\Delta V_{\mathrm{C}}=\Delta h_{\mathrm{S}} V_{\mathrm{S}}+\Delta h_{\mathrm{G}} V_{\mathrm{G}},
$$

where, $h$ denotes the current division factor (CDF), and $h_{\mathrm{S}}$ and $h_{\mathrm{G}}$ correspond to the CDFs of the signal line and guard trace, respectively. The CDF is defined as the ratio of common-mode current flowing along the line of interest to the entire common-mode current, and indicates the degree of imbalance. The difference in $\mathrm{CDFs}, \Delta h$, takes a value other than 0 at the interface where the cross-sectional structure changes.

In Ref. [5], the reduction was calculated without taking into consideration the second term on the right-hand side in Eq. (2). If the guard-trace voltage is quite low, the common-mode source due to the guard trace is negligible. When the guard-trace voltage is high, however, the

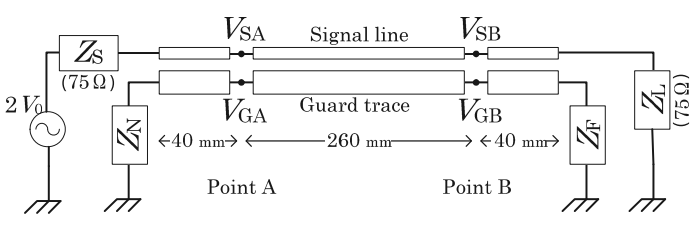

(a) Coupled transmission lines treated in SPICE simulation.

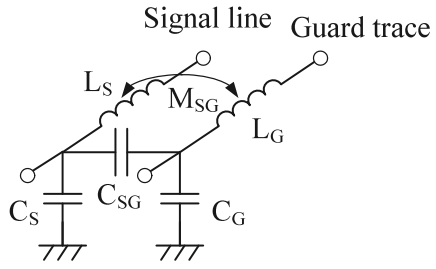

(b) Unit cell of coupled-transmission-line model.

Fig. 5 Coupled-transmission-line model of signal and guard traces.

Table 1 Value of elements in Fig. 5(b).

\begin{tabular}{|c|c||c|c|}
\hline$L_{\mathrm{S}}$ & $550 \mathrm{nH} / \mathrm{m}$ & $C_{\mathrm{S}}$ & $41 \mathrm{p} \mathrm{F} / \mathrm{m}$ \\
\hline$L_{\mathrm{G}}$ & $470 \mathrm{nH} / \mathrm{m}$ & $C_{\mathrm{G}}$ & $55 \mathrm{p} \mathrm{F} / \mathrm{m}$ \\
\hline$M_{\mathrm{SG}}$ & $240 \mathrm{nH} / \mathrm{m}$ & $C_{\mathrm{SG}}$ & $30 \mathrm{p} \mathrm{F} / \mathrm{m}$ \\
\hline
\end{tabular}

common-mode voltage source increases and generates large emissions. Therefore, we need to assess the guard-trace voltage at the changing point of the cross-sectional structure.

These voltages could be evaluated with the SPICE circuit simulator because the guard-trace voltage was caused by crosstalk. The calculation model for the coupled transmission lines in Fig. 5(a) was expressed as an LC ladder in the SPICE simulation. The unit cell of the LC ladder is outlined in Fig. 5(b). Table 1 summarizes the values of elements composed of the unit cell. The length of the unit cell was $10 \mathrm{~mm}$, which corresponds to about $1 / 17$ of the wavelength at $1 \mathrm{GHz}$.

In Fig. 5(a), $V_{\mathrm{SA}}, V_{\mathrm{GA}}, V_{\mathrm{SB}}$, and $V_{\mathrm{GB}}$ denote the normal mode voltages, respectively. The first subscripts of ' $S$ ' and ' $G$ ' denote the signal line and guard trace, respectively. The second subscripts ' $\mathrm{A}$ ' and ' $\mathrm{B}$ ' denote the positions on the lines.

\subsection{Validation of Proposed Method}

\subsubsection{Criterion}

This paper uses a criterion on the effect of the guard trace, i.e., the common-mode source voltage with the guard trace must be less than that without the guard trace to avoid an increase in radiation. Now, let us focus on the commonmode source voltage at point $\mathrm{B}$ in the test board, as shown in Fig. 2. According to Eq. (A. 3),

$$
\left|\Delta V_{\mathrm{C}}^{\prime}\right|>\left|\Delta V_{\mathrm{CS}}+\Delta V_{\mathrm{CG}}\right|,
$$

where the prime indicates the reference board (without the guard trace). Using the general relationship between two arbitrary complex numbers, 
Table 2 Comparison of CDFs.

\begin{tabular}{|l|l|c|c|c|}
\hline PCB & Line & $h_{\mathrm{a}}$ & $h_{\mathrm{b}}$ & $\Delta h$ \\
\hline Reference & Signal line & 0.157 & 0.014 & 0.143 \\
\hline Test board & Signal line & 0.067 & 0.010 & 0.057 \\
\cline { 2 - 5 } & Guard trace & 0.165 & 0.010 & 0.155 \\
\hline
\end{tabular}

Table 3 Termination conditions for guard trace.

\begin{tabular}{|l|c|c|c|c|c|c|}
\hline$\Gamma$ & -1 & -0.9 & -0.5 & 0 & 0.5 & 0.9 \\
\hline$Z(\Omega)$ & 0 & 3.3 & 20 & 68 & 200 & 1200 \\
\hline
\end{tabular}

$$
\left|\Delta V_{\mathrm{CS}}\right|+\left|\Delta V_{\mathrm{CG}}\right| \geq\left|\Delta V_{\mathrm{CS}}+\Delta V_{\mathrm{CG}}\right|
$$

the sufficient condition for Eq. (3) to hold is given as

$$
\left|\Delta V_{\mathrm{C}}^{\prime}\right|>\left|\Delta V_{\mathrm{CS}}\right|+\left|\Delta V_{\mathrm{CG}}\right| \text {. }
$$

The signal line stays approximately at the same voltage independently of the guard trace.

$$
\left|\Delta h^{\prime} V_{\mathrm{S}}\right|>\left|\Delta h_{\mathrm{S}} V_{\mathrm{S}}\right|+\left|\Delta h_{\mathrm{G}} V_{\mathrm{G}}\right|
$$

We can derive the following condition in the end:

$$
\frac{\left|V_{\mathrm{G}}\right|}{\left|V_{\mathrm{S}}\right|}<\frac{\left|\Delta h^{\prime}\right|-\left|\Delta h_{\mathrm{S}}\right|}{\left|\Delta h_{\mathrm{G}}\right|}
$$

Table 2 lists the CDFs of the test board in Fig. 2 and the reference board. The CDFs were calculated according to Ref. [9]. As a result, the criterion on the effect of the guard trace in this paper is given as

$$
\begin{aligned}
\frac{\left|V_{\mathrm{G}}\right|}{\left|V_{\mathrm{S}}\right|} & <\frac{\left|\Delta h^{\prime}\right|-\left|\Delta h_{\mathrm{S}}\right|}{\left|\Delta h_{\mathrm{G}}\right|} \\
& =\frac{0.143-0.057}{0.155}=0.55(-5.1 \mathrm{~dB})
\end{aligned}
$$

From Eq. (8), $V_{\mathrm{GB}}$ must be less than $V_{\mathrm{SB}}$ by about $5 \mathrm{~dB}$. Since the source voltage, $2 V_{0}$, in this calculation is $106 \mathrm{~dB} \mu \mathrm{V}$, the signal voltage $V_{\mathrm{SB}}$ becomes approximately $100 \mathrm{~dB} \mu \mathrm{V}$. Based on the above discussion, at $V_{\mathrm{GB}}$ over $95 \mathrm{~dB} \mu \mathrm{V}$, the common-mode radiation caused by the guardtrace voltage can be higher than that without a guard trace.

The changes in the cross-sectional structure at points A and B in Fig. 2 are symmetrical to the $y z$ plane. Therefore, the differences $\Delta h\left(=h_{\mathrm{a}}-h_{\mathrm{b}}\right)$ at points $\mathrm{A}$ and $\mathrm{B}$ have the same magnitude and the opposite sign. That is, the criteria given by Eq. (8) at both points A and B are the same.

The voltages on the guard trace under various combinations of $Z_{\mathrm{N}}$ and $Z_{\mathrm{F}}$ are examined in the following sections. First, the values of the far-end termination resistor are varied with a short termination at the near end. Next, the way the termination resistors are arranged are evaluated under matched termination.

\subsubsection{Termination Impedance}

We used several termination resistors that corresponded to the voltage reflection coefficients $(\Gamma)$ listed in Table 3 . The characteristic impedance of the guard trace is approximately

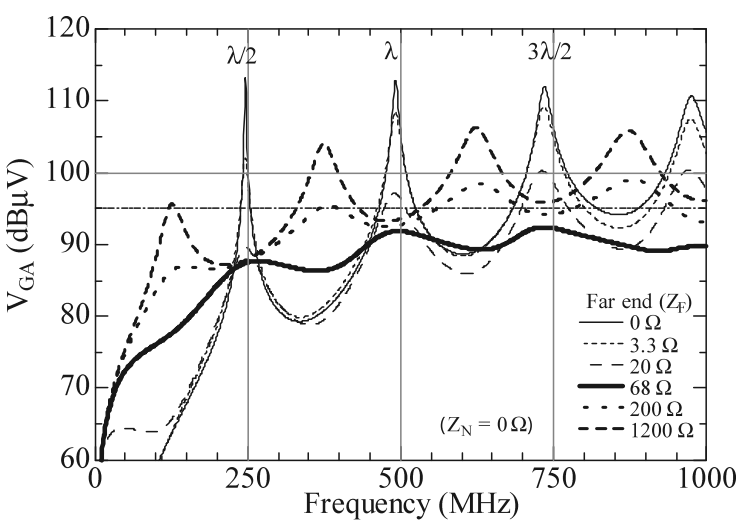

(a) At point A.

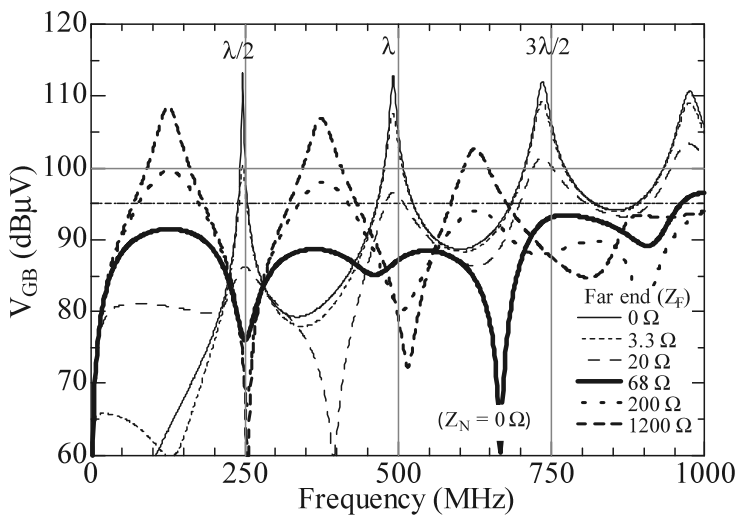

(b) At point B.

Fig. 6 Calculated guard-trace voltages with different termination impedances.

$65 \Omega$.

Figures 6(a) and 6(b) plot $V_{\mathrm{GA}}$ and $V_{\mathrm{GB}}$, respectively. When $Z_{\mathrm{F}}$ is matched $(68 \Omega)$, both $V_{\mathrm{GA}}$ and $V_{\mathrm{GB}}$ stay at less than $95 \mathrm{~dB} \mu \mathrm{V}$ almost over the entire frequency range. When $Z_{\mathrm{F}}$ is less than $68 \Omega$, the guard trace behaves like a transmission line with short termination at both ends. Therefore, both $V_{\mathrm{GA}}$ and $V_{\mathrm{GB}}$ increase at the resonant frequencies given in Eq. (1). With the increase in $Z_{\mathrm{F}}$, the resonant voltage decreases. When $Z_{\mathrm{F}}$ is greater than $68 \Omega$, both $V_{\mathrm{GA}}$ and $V_{\mathrm{GB}}$, in contrast, increase at frequencies corresponding to resonances of $\lambda / 4,3 \lambda / 4 \ldots$ due to the open and short terminations at each end. Thus, the resonant voltage increases due to mismatching.

\subsubsection{Arrangement of Termination Resistors}

This section discusses the arrangement of the termination resistors with the matched termination. The termination of interest was matched in the experiments and the rest were shortened. Figures 7(a) and 7(b) plot $V_{\mathrm{GA}}$ and $V_{\mathrm{GB}}$, respectively. The "both ends" and "far end" cases in Fig. 7 satisfy the condition of "less than $95 \mathrm{~dB} \mu \mathrm{V}$ " given in Eq. (8), almost over the entire frequency range. The "both ends" case is not necessarily superior to the "far end" case. Therefore, the "far end" case has an advantage in reduced fabrication 


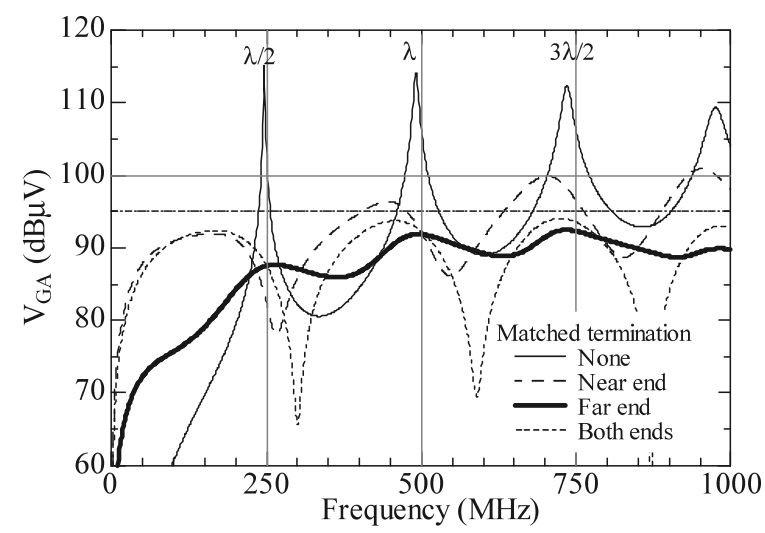

(a) At point A.

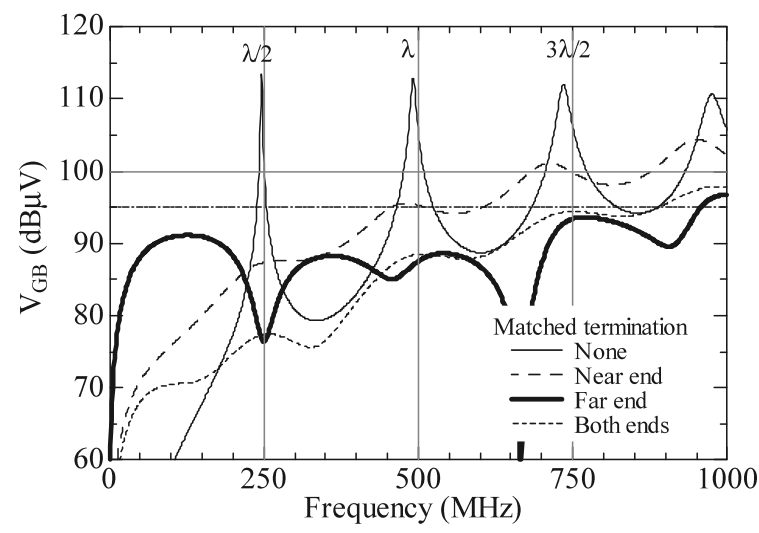

(b) At point B.

Fig. 7 Calculated guard-trace voltages with arrangement of termination resistors.

cost because the number of resistors can be reduced by half. Although the "near end" case also mitigates resonance, both $V_{\mathrm{GA}}$ and $V_{\mathrm{GB}}$ are larger than those of the "far end" case. This is because the far-end crosstalk is larger than near-end crosstalk. As a result, the most cost-effective arrangement is to shorten $Z_{\mathrm{N}}(0 \Omega)$ and match $Z_{\mathrm{F}}(68 \Omega)$.

\section{Measurement of Common-Mode Radiation}

\subsection{Measurement Setup}

The radiation from the test board was measured in a semianechoic chamber. The board and the receiving antenna were fixed $1 \mathrm{~m}$ above the floor, as shown in Fig. 8. The $y$ axis of the board was vertically directed. The source signal of $100 \mathrm{~dB} \mu \mathrm{V}$ was fed through a coaxial cable from the tracking generator of a spectrum analyzer. The maximum radiation was measured while rotating the test board on a turntable.

\subsection{Termination Impedance}

Figure 9(a) plots the measured results when only the resistors in far-end termination were varied. The difference in radiation from that of the reference board, which has no guard

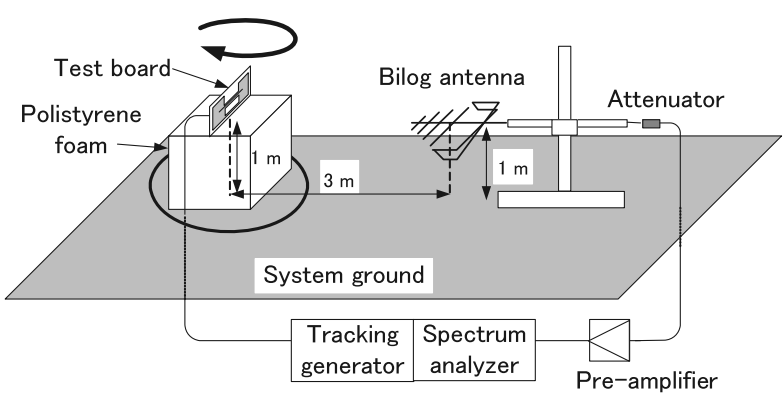

Fig. 8 Setup for measuring radiation.

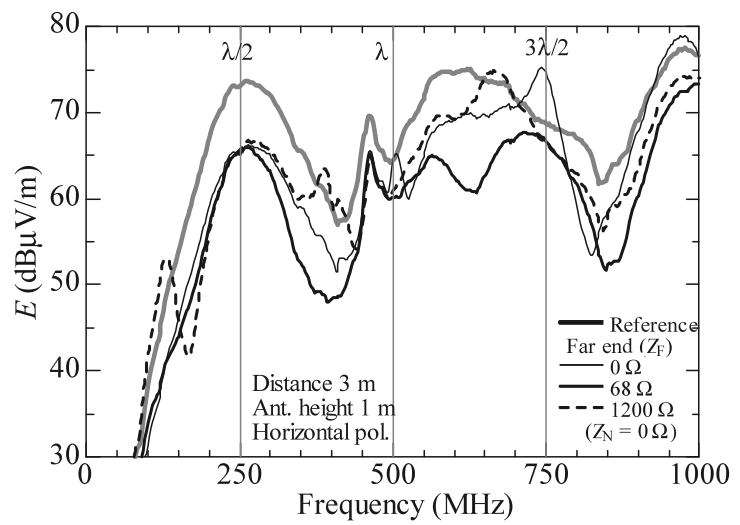

(a) Magnitude of radiation.

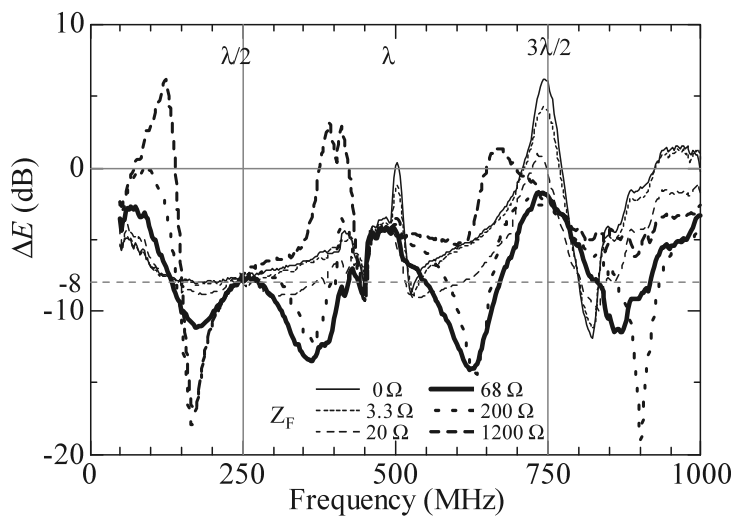

(b) Reduction due to guard trace.

Fig. 9 Comparison of radiated emissions among different termination resistors implemented on guard trace.

trace, is shown in Fig. 9(b). The difference means reduced radiation due to the guard trace.

Figures 6 and 9(b) indicate that there is a good relationship between the guard-trace voltage and radiation other than for the resonant frequency of $250 \mathrm{MHz}$. The total radiation is given as a vector sum of radiated emissions from all common-mode sources and depends on the phase relationship between these sources. At $250 \mathrm{MHz}$, the radiation caused by common-mode sources due to $V_{\mathrm{GA}}$ and $V_{\mathrm{GB}}$ are canceled out owing to the symmetry of the test board used in the measurement and no increase in EMI due to guard-trace resonance occurs.

The radiation in matched termination is suppressed at 


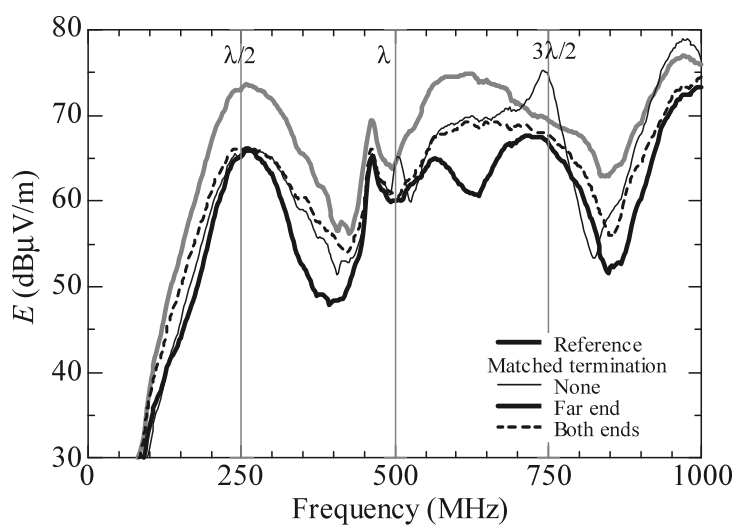

(a) Magnitude of radiation.

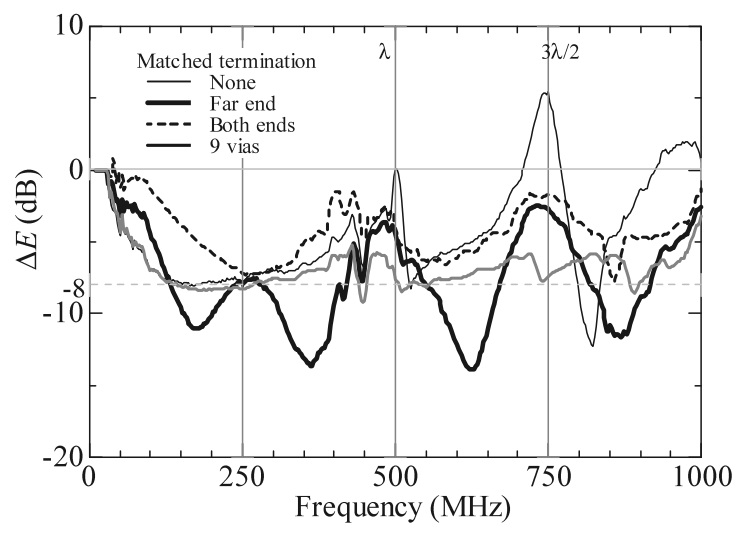

(b) Reduction due to guard trace.

Fig. 10 Comparison of radiated emissions at different locations of termination resistors.

almost all frequencies of interest. This results from the suppression of resonance in the guard trace. We found that radiation in unmatched termination increased at the resonant frequencies.

\subsection{Arrangement of Termination Resistors}

Figure 10(a) plots the measured results when the arrangements of the termination resistors were varied. Also, the difference in radiation from that of the reference board is shown in Fig. 10(b). Figure 10(b) indicates that in both the "far end" and "both ends" cases, the increases caused by guard-trace resonance were suppressed.

In addition, Fig. 10(b) also shows a case with "nine vias," in which seven additional vias were added at the same intervals in the guard trace in addition to the short terminations at both ends. The lowest resonant frequency of the guard trace is about $2.0 \mathrm{GHz}$. Therefore, because the voltage of the guard trace at any point stays much lower than $95 \mathrm{~dB} \mu \mathrm{V}$, the reduction in radiation reached the calculated value $(-8 \mathrm{~dB})$ over the entire frequency range.

The difference from the "nine vias" case suggests the effect of guard-trace voltage. In the "far end" case, a reduction of more than the calculated value of $-8 \mathrm{~dB}$ can be observed because the reduction depends on the phase dif- ference in the voltages between the signal and guard traces. Therefore, although the guard-trace resonance is suppressed more than that in the "no (short) termination" case, the slight effect caused by low guard-trace voltage remains.

\section{Conclusion}

We proposed a novel technique of eliminating vias on the guard trace without increasing radiation. Instead of vias with short intervals, matching at both ends of the guard trace was assessed to suppress resonance and reduce commonmode radiation. This approach was derived from the viewpoint of the guard trace acting as a transmission line. This method was tested to verify its efficiency with a circuit simulation of guard-trace voltages and by measuring radiation from a test board. The values and the arrangements of termination resistors were investigated in detail. As a result, we found that the proposed approach could remain at low guard-trace voltages and avoid increases in radiation. In addition, we also demonstrated that matched termination at the far end of the guard trace could sufficiently suppress guardtrace resonance at all frequencies. We eventually found that at least two vias at both ends of the guard trace and only one matching resistor at the far end could suppress guard-trace resonance. With respect to fewer vias, the new method has the advantage of reducing restrictions on the PCB layout at the design stage.

\section{Acknowledgments}

We would like to thank Ms. Yuko Fukutsuka, an undergraduate at Okayama University, for her conscientious efforts with the overall measurements and calculations.

\section{References}

[1] D.M. Hockanson, J.L. Drewniak, T.H. Hubing, T.P. Van Doren, F. Sha, and M.J. Wilhelm, "Investigation of fundamental EMI source mechanisms driving common-mode radiation from printed circuit boards with attached cables," IEEE Trans. Electromagn. Compat., vol.38, no.4, pp.557-566, Nov. 1996.

[2] F.B. Leferink, "Reduciton of printed circuit board radiated emission," Proc. IEEE Int. Symp. Electromagnetic Compatibility, pp.431-438, Austin, TX, USA, Aug. 1997.

[3] T. Watanabe, O. Wada, T. Miyashita, and R. Koga, "Common-modecurrent generation caused by difference of unbalance of transmission lines on a printed circuit board with narrow ground pattern," IEICE Trans. Commun., vol.E83-B, no.3, pp.593-599, March 2000.

[4] T. Watanabe, O. Wada, A. Namba, K. Fujimori, S. Matsunaga, and R. Koga, "Quantitative evaluation of common-mode radiation from a PCB based on imbalance difference model," Proc. Int. Symp. Electromagnetic Compatibility, pp.201-204, Sendai, Japan, June 2004.

[5] T. Matsushima, T. Watanabe, Y. Toyota, R. Koga, and O. Wada, "Evaluation of EMI reduction effect of guard traces based on imbalance difference model," IEICE Trans. Commun., vol.E92-B, no.6, pp.2193-2200, June 2009.

[6] R.W. Dockey and R.F. German, "New techniques for reducing printed circuit board common-mode radiation," Proc. IEEE Int. Symp. Electromagnetic Compatibility, pp.334-339, Dallas, TX, USA, Aug. 1993.

[7] D.S. Britt, D.M. Hockanson, F. Sha, J.L. Drewniak, T.H. Hubing, 
and T.P. Van Doren, "Effects of gapped groundplanes and guard traces on radiated EMI," Proc. IEEE Int. Symp. Electromagnetic Compatibility, pp.159-164, Austin, TX, USA, Aug. 1997.

[8] Y. Kayano, M. Tanaka, J.L. Drewniak, and H. Inoue, "Commonmode current due to a trace near a PCB edge and its suppression by a guard band," IEEE Trans. Electromagn. Compat., vol.46, no.1, pp.46-53, Feb. 2004.

[9] T. Matsushima, T. Watanabe, Y. Toyota, R. Koga, and O. Wada, "Increase of common-mode radiation due to guard trace voltage and determination of effective via-location," IEICE Trans. Commun. vol.E92-B, no.6, pp.1929-1936, June 2009.

[10] S. Bokhari and H. Ali, "On grounded co-planar waveguides as interconnects for $10 \mathrm{~GB} / \mathrm{s}$ signals," Proc. IEEE Int. Symp. Electromagnetic Compatibility, pp.607-609, Aug. 2003.

[11] T. Watanabe, H. Fujihara, O. Wada, R. Koga, and Y. Kami, "A prediction method of common-mode excitation on a printed circui board having a signal trace near the ground edge," IEICE Trans. Commun., vol.E87-B, no.8, pp.2327-2334, Aug. 2004.

\section{Appendix: Common-Mode Excitation in Two Trans- mission Lines}

In Ref. [9], we derived common-mode excitation in threeconductor transmission lines with the imbalance difference model. The results were applied to the current case in the following way.

Figure A-1(a) shows the three-conductor transmission lines that contain a signal line, a guard trace, and a return plane. These transmission lines consist of two parts. In part $\mathrm{A}$, the transmission lines have a narrow return plane. However, the return plane in part $\mathrm{B}$ is wider than that in part $\mathrm{A}$.

Figure $\mathrm{A} \cdot 1(\mathrm{~b})$ is a potential diagram indicating the real and virtual potentials. $V_{\mathrm{pS}}, V_{\mathrm{pG}}$, and $V_{\mathrm{pR}}$ correspond to the real potentials of the signal line, the guard trace, and the

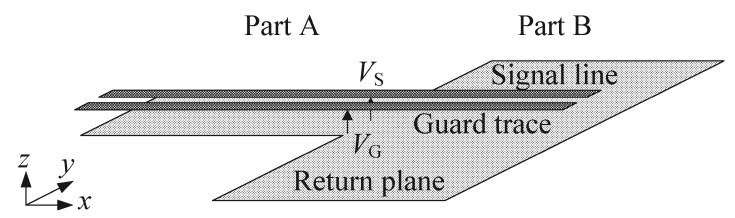

(a) Multi-conductor transmission lines with interface where width of return plane changes.

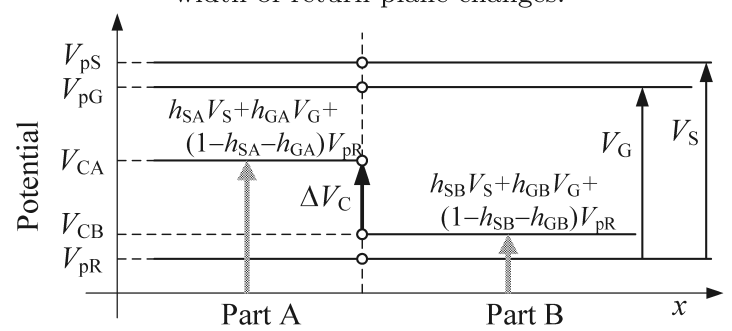

(b) Potential diagram around interface.

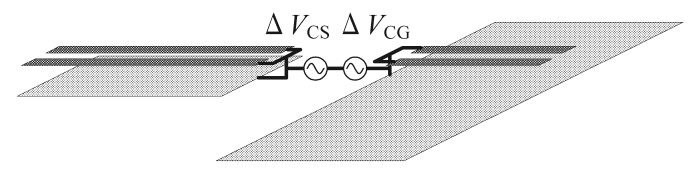

(c) Common-mode excitation sources.

Fig. A. 1 Imbalance difference model of microstrip with two signal lines and ground plane. return plane, respectively. $V_{\mathrm{Ca}}$ and $V_{\mathrm{Cb}}$ denote the virtual potentials in parts $\mathrm{A}$ and $\mathrm{B}$, respectively. Also, $V_{\mathrm{S}}$ and $V_{\mathrm{G}}$ correspond to the voltage of the signal line and the guard trace to the return plane, respectively.

To evaluate common-mode generation, we used a current division factor (CDF) [3], which represents the degree of imbalance. In Fig. A. 1(a), the CDF of the signal line, $h_{\mathrm{S}}$, has been calculated by regarding the guard trace and return plane as return paths [4]. The CDF of the guard trace, $h_{\mathrm{G}}$, on the other hand, has been calculated by regarding the signal line and return plane as return paths. Then, the virtual common-mode potentials, $V_{\mathrm{Ca}}$ and $V_{\mathrm{Cb}}$, are given as

$$
\begin{aligned}
& V_{\mathrm{Ca}}=h_{\mathrm{Sa}} V_{\mathrm{pS}}+h_{\mathrm{Ga}} V_{\mathrm{pG}}+\left(1-h_{\mathrm{Sa}}-h_{\mathrm{Ga}}\right) V_{\mathrm{pR}}, \\
& V_{\mathrm{Cb}}=h_{\mathrm{Sb}} V_{\mathrm{pS}}+h_{\mathrm{Gb}} V_{\mathrm{pG}}+\left(1-h_{\mathrm{Sb}}-h_{\mathrm{Gb}}\right) V_{\mathrm{pR}},
\end{aligned}
$$

respectively. The difference in common-mode potentials between parts A and B is given as

$$
\begin{aligned}
\Delta V_{\mathrm{C}} & =V_{\mathrm{Cb}}-V_{\mathrm{Ca}} \\
& =\left(h_{\mathrm{Sb}}-h_{\mathrm{Sa}}\right) V_{\mathrm{S}}+\left(h_{\mathrm{Gb}}-h_{\mathrm{Ga}}\right) V_{\mathrm{G}} \\
& =\Delta h_{\mathrm{S}} V_{\mathrm{S}}+\Delta h_{\mathrm{G}} V_{\mathrm{G}} \\
& =\Delta V_{\mathrm{CS}}+\Delta V_{\mathrm{CG}},
\end{aligned}
$$

where $\Delta h_{\mathrm{S}}$ and $\Delta h_{\mathrm{G}}$ correspond to the CDF difference in the signal line and the guard trace, respectively. We found from Eq. (A.3) that the total common-mode voltage source is the vector sum of common-mode voltage sources on the signal line and the guard trace. We eventually illustrated the common-mode voltage sources shown in Fig. A. 1(c) at the interface where the cross section of the multi-conductor transmission lines changes.

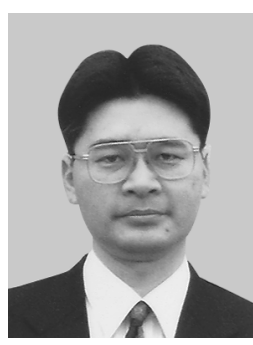

Tetsushi Watanabe was born in Okayama, Japan, on May 11, 1967. He received his B.E., M.E. and Ph.D. degrees in Electronics Engineering from Okayama University, Japan, in 1990, 1992 and 2004, respectively. Since 1992, he has been with the Industrial Technology Center of Okayama Prefecture. He has engaged in a study of immunity on LAN cables, measurement systems of EMC, and EMI reduction techniques. $\mathrm{He}$ is a member of the Japan Institute of Electronics Packaging.

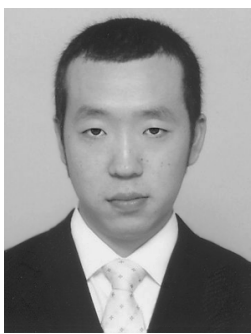

Tohlu Matsushima was born in Hyogo, Japan, on May 19, 1982. He received his M.E. and Ph.D. degrees in Communication Network Engineering from Okayama University, Japan, in 2006 and 2009, respectively. He is currently an Assistant Professor at Kyoto University. His research interest is in the electromagnetic interference problems. He is a member of IEEE and the Japan Institute of Electronics Packaging. 


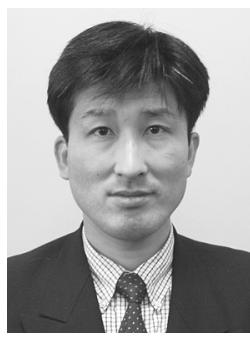

Yoshitaka Toyota was born in Okayama, Japan, on September 17, 1968. He received his $\mathrm{Ph} . \mathrm{D}$. degree in electronic engineering from Kyoto University, Japan, in 1996. From 1996 to 1998, he was with Yokogawa Electric Co., Ltd. and in 2005 he worked at Georgia Tech as an overseas research scholar of the Ministry of Education, Culture, Sports, Science and Technology (MEXT) of Japan. He is currently an Associate Professor at Okayama University. His research interest includes EMC design in highspeed digital systems. He is a member of IEEE, the Japan Institute of Electronics Packaging, and the Japan Society of Applied Physics.

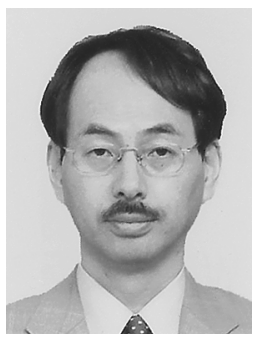

Osami Wada was born in 1957. He received the B.E., M.E. and Dr.E. degrees in electronics from Kyoto University, Japan, in 1981, 1983 and 1987, respectively. Between 1988 and 2005 he worked at the Faculty of Engineering of Okayama University, Japan. Since 2005 he has been a Professor at the Department of Electrical Engineering at the Graduate School of Engineering of Kyoto University, Japan. He is a member of IEE of Japan, the Japan Institute of Electronics Packaging, and IEEE.

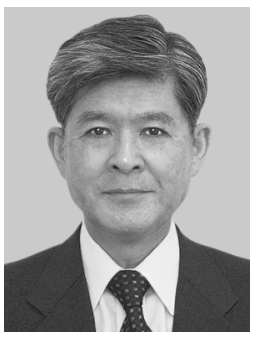

Ryuji Koga was born in Tokyo on 1, January 1945. He received the B.E., M.E. and D.E. degrees from Kyoto University in 1967, 1969 and 1972, respectively. He was first with the Atomic Energy Institute of Kyoto University. In 1976 he moved to Okayama University as a lecturer, and started studies on electronics. On the way of developing the laser-sensing system, he was suffered from EMI from digital system attached to the extremely sensitive analog system for the laser sensing system; since then he has been engaged in the study of EMC problem. He is now Professor of Okayama University, and had been the chairperson of the Technical Committee of The Institute of Electronics, Information and Communication Engineers, JAPAN. He had also been the chair of IEEE EMCS Japan chapter, and chaired the international symposium EMC'09/Kyoto. He is presently the chair of URSI-E Committee, Japan. He experienced the director of the foreign student center of Okayama University, as well as a member of the board of trustee, Okayama University. 